DOI: https://doi.org/10.46296/yc.v5i9edespag.0097

\title{
ESTRATEGIAS DE MARKETING PARA LOS PRODUCTOS AGRÍCOLAS Y SU RELACIÓN CON LA PRODUCTIVIDAD EN LAS BODEGAS COMERCIALES DEL CANTON JIPIJAPA
}

\section{MARKETING STRATEGIES FOR AGRICULTURAL PRODUCTS AND THEIR RELATIONSHIP WITH PRODUCTIVITY IN THE COMMERCIAL WAREHOUSES OF THE JIPIJAPA CANTON}

\author{
Ross-Medranda Cesar Giovanny ${ }^{1}$; Tamayo-Cevallos Christian David ${ }^{2}$ \\ ${ }^{1}$ Estudiante de la Maestría en Administración de Empresas, Instituto de Posgrado, \\ Grupo de Investigación: Entorno Empresarial, Universidad Técnica de Manabí. \\ Portoviejo Ecuador. cross3888@utm.edu.ec \\ ${ }^{2}$ Facultad de Ciencias Administrativas y Económicas de la Universidad Técnica de \\ Manabí, Grupo de Investigación: Entorno Empresarial, Universidad Técnica de \\ Manabí. Portoviejo Ecuador.christian.tamayo@utm.edu.ec
}

\begin{abstract}
Resumen
La producción agrícola puede considerarse como la columna vertebral del comercio en Manabí. Más del $60 \%$ de los trabajadores rurales laboran en el sector agrícola. La agricultura se refiere al cultivo de la tierra y la cría de animales y plantas para proporcionar alimentos, plantas medicinales con fibra y otros productos para sustentar la calidad de vida. El rol del sector agrícola varía considerablemente cuando la urbanización y la migración laboral en la economía están invitando a mayores oportunidades para que los trabajadores agrícolas sostengan los niveles de producción, enfatizando así la transformación del sector de mano de productores agrícolas y agro-emprendedores. La materia prima generada por Jipijapa se concentra en gran medida en los productos agrícolas como maíz, naranja, café, yuca, y en un segundo orden banano, arroz, maní entre otros. En adición a esto, productos como la ganadería, avicultura y pesca también están aportando de forma significativa a la economía local de manera creciente. Bajo esta premisa, la presente investigación tiene la intención de discernir en las estrategias de marketing más óptimas para fomentar la productividad en las bodegas comerciales del cantón Jipijapa.
\end{abstract}

Palabras claves: producción agrícola, producción, marketing, bodegas comerciales.

\begin{abstract}
Agricultural production can be considered as the backbone of commerce in Manabí. More than $60 \%$ of rural workers work in the agricultural sector. Agriculture refers to the cultivation of the land and the raising of animals and plants to provide food, medicinal plants with fiber and other products to support the quality of life. The role of the agricultural sector varies considerably when urbanization and labor migration in the economy are inviting greater opportunities for agricultural workers to sustain production levels, thus emphasizing the transformation of the sector at the hands of agricultural producers and agro-entrepreneurs. The raw material generated by Jipijapa is largely concentrated in agricultural products such as corn, orange, coffee, cassava, and in a second order, bananas, rice, and peanuts, among others. In addition to this, products such as livestock, poultry and fishing are also contributing significantly to the local economy in an increasing way. Under this premise, the present research intends to discern the most optimal marketing strategies to promote productivity in commercial warehouses in the Jipijapa City.
\end{abstract}

Keywords: agricultural production, production, marketing, commercial warehouses.

Información del manuscrito:

Fecha de recepción: 28 de junio de 2021.

Fecha de aceptación: 31 de julio de 2021.

Fecha de publicación: 04 de agosto de 2021. 


\section{Introducción}

La producción agrícola puede considerarse como la columna vertebral del comercio en Manabí. Más del $60 \%$ de los trabajadores rurales laboran en el sector agrícola. La agricultura se refiere al cultivo de la tierra y la cría de animales y plantas para proporcionar alimentos, plantas medicinales con fibra y otros productos para sustentar y mejorar la vida. La agricultura fue el desarrollo clave en el surgimiento de la civilización humana sedentaria, mediante la cual el cultivo de especies domesticadas creó excedentes de alimentos que permitieron a las personas vivir en ciudades (Bhosage, Mr, 2018).

El rol del sector agrícola varía considerablemente cuando la urbanización y la migración laboral en la economía están invitando a mayores oportunidades para que los trabajadores agrícolas sostengan los niveles de producción, enfatizando así la transformación del sector de mano de productores agrícolas y agro-emprendedores.

En términos generales, Las mujeres desempeñan un papel fundamental en la agricultura de los países en desarrollo. En ese sentido, el porcentaje de mujeres que dependen de la agricultura llega al $84 \%$. Las mujeres representan aproximadamente el $33 \%$ de los agricultores y el $47 \%$ de los trabajadores agrícolas (Das, Sreelakshmi, 2019). Aunque las mujeres están muy involucradas en la agricultura en pequeña escala, su papel rara vez se reconoce 0 recompensa por completo. Están involucrados en una variedad de estrategias de subsistencia, pero su contribución a la agricultura se considera simplemente como una "ayuda" o un trabajo de subsistencia no remunerado. A pesar de su posición dominante en la fuerza laboral, las mujeres indias aún enfrentan desventajas extremas en términos de salarios, derechos sobre la tierra, acceso al crédito y representación en las organizaciones de agricultores locales. (Das, Sreelakshmi, 2019).

En línea con otros sectores, el comercio minorista ha adoptado el marketing electrónico o el marketing en Internet, ampliando el alcance a los clientes más allá de sus lugares de compra convencionales. Los agricultores pueden utilizar Internet 
de muchas formas posibles para vender sus productos (Razorvin, Ivan \& Usova, N. \& Loginov, Mihail, 2021). El uso de Internet como una forma de vender productos agrícolas está cambiando los canales de comercialización en el sector agroindustrial (Krizhanovskaya, 2014). Los mercados agrícolas se caracterizan por una escasa competitividad, fragmentación, ineficiencia, presencia de intermediarios ejecutivos $y$ frecuentes manipulaciones de precios (Ploeg, Jan, 2019).

La materia prima generada por Jipijapa se concentra en gran medida en los productos agrícolas como maíz, naranja, café, yuca, y en un segundo orden banano, arroz, maní entre otros. De forma similar, productos como la ganadería, avicultura y pesca también están aportando de forma significativa a la economía local de manera creciente. (Bose, Subhas Chandra \& Kiran, Ravi, 2021). Bajo esta premisa, la presente investigación tiene la intención de discernir en las estrategias de marketing más óptimas para fomentar la productividad en las bodegas comerciales del cantón Jipijapa.

\section{Metodología}

El proceso metodológico se desarrolló bajo el paradigma de enfoque mixto, para lo cual se utilizará información estadística de fuentes confiables como departamentos gubernamentales etc. los cuales representan la realidad sobre la productividad y las principales estrategias de marketing que puedan agilizar y fomentar la productividad de las bodegas comerciales del cantón Jipijapa.

Por otro lado, para realizar procedimientos de investigación, en primera instancia se desarrolló un proceso de revisión literaria para enunciar teorías que sustentan la investigación de fenómenos y procesos, y luego un proceso típico de investigación de campo. La tecnología de campo permite la observación del proceso en contacto directo con el objeto de investigación, y la recolección de información que involucra a la teoría y práctica en busca de información significativa y precisa. Los criterios para seleccionar la muestra de investigación se determinan en función de la duración del servicio. 


\subsection{Tipo de investigación}

La presente investigación es de tipo descriptiva, dado que se pretendió caracterizar cada una de las variables en estudio, tal como se presentan en la realidad estudiada. Además de ser de tipo aplicada, tomando en cuenta que se pretende generar una solución a la realidad encontrada con el fin de proponer estrategias de marketing para fomentar la productividad en las bodegas comerciales del cantón Jipijapa.

\subsection{Población objeto de estudio}

Posterior a la revisión de los datos estadísticos del Sistema nacional de información (SNI, 2015), se pudo constatar que el cantón Jipijapa posee 110 registros de bodegas comerciales. Con base a ello se realizó un muestreo intencionado, teniendo como criterios para la selección de la muestra bodegas comerciales enfocadas en el sector agrícola.

\subsection{Técnicas de recolección de la información}

Para la recolección de información se utilizaron técnicas de encuestas y entrevistas estructuradas como herramientas para comprender las características del sector agrícola de Jipijapa, especialmente los factores que pueden interferir con la productividad del sector comercial. En cuanto a las herramientas, las preguntas son mixtas, con opciones sí y no para determinar finalmente las opiniones de los trabajadores agrícolas sobre la implementación de estrategias de marketing basadas en escenarios actuales.

\section{Resultados}

Tabla 1. Estrategias publicitarias de las bodegas comerciales de Jipijapa.

\begin{tabular}{|c|c|c|c|c|c|c|c|}
\hline \multirow[b]{2}{*}{ 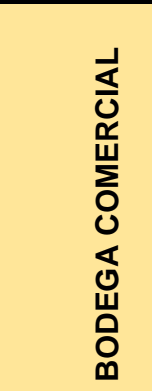 } & \multicolumn{5}{|c|}{ ESTRATEGIAS PUBLICITARIAS UTILIZADAS } & \multicolumn{2}{|c|}{$\begin{array}{c}\text { OTRAS } \\
\text { ESTRATEGIAS DE } \\
\text { VENTAS UTILIZADAS } \\
\end{array}$} \\
\hline & c & 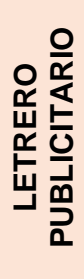 & 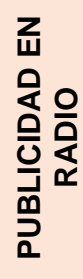 & 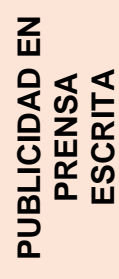 & 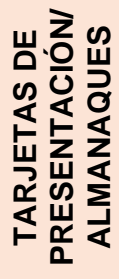 & 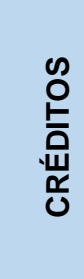 & 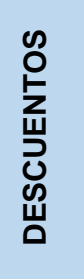 \\
\hline $\begin{array}{l}\text { Comercial } \\
\text { Muñiz }\end{array}$ & $X$ & $X$ & & & $X$ & $X$ & $X$ \\
\hline
\end{tabular}




\begin{tabular}{|c|c|c|c|c|c|c|}
\hline $\begin{array}{l}\text { Comercial } \\
\text { Marón } \\
\text { Villamar }\end{array}$ & $x$ & $X$ & & $x$ & $x$ & $x$ \\
\hline $\begin{array}{l}\text { Comercial } \\
\text { Joffre Pérez }\end{array}$ & & & & & $x$ & $X$ \\
\hline $\begin{array}{l}\text { Comercial } \\
\text { Ross } \\
\text { Medranda }\end{array}$ & $x$ & & & $x$ & $X$ & $x$ \\
\hline $\begin{array}{l}\text { Comercial } \\
\text { Castillo Nieto }\end{array}$ & & $X$ & $x$ & & $X$ & $X$ \\
\hline $\begin{array}{l}\text { Comercial } \\
\text { Caracol }\end{array}$ & $X$ & $X$ & & $x$ & $x$ & $x$ \\
\hline $\begin{array}{l}\text { Comercial } \\
\text { Bustamante }\end{array}$ & $x$ & & & $x$ & $X$ & $X$ \\
\hline $\begin{array}{l}\text { Comercial } \\
\text { Jandry } \\
\text { Villamar }\end{array}$ & $x$ & & & $x$ & $X$ & $X$ \\
\hline
\end{tabular}

Fuente: Autoría propia.

En base a las encuestas realizadas a las bodegas comerciales enfocadas en la producción agrícola del cantón Jipijapa, cuyas preguntas se encontraron orientadas a conocer la opinión de los comerciantes en cuanto a la implementación de estrategias de marketing con la finalidad de mejorar la productividad de las bodegas comerciales, se puede discernir que el $70 \%$ de los emprendedores consideran que las estrategias de marketing digital podrían fomentar la productividad de las bodegas comerciales de cara al panorama actual. Sin embargo, el $30 \%$ de comerciantes aseguran que la ejecución de dichas estrategias por parte de la iniciativa gubernamental y municipal se encuentra limitada por la escasez de fondos y la ínfima cantidad de proyectos privados para promover nuevas estrategias de marketing en las bodegas comerciales de la agroindustria en la ciudad de jipijapa, y de cara al panorama actual, se determinó que El marketing digital representa una tentativa viable para fomentar la productividad comercial. 
Gráfico 1. Grafica de ventas.

\section{VENTAS AÑO 2020 EN DÓLARES}

$\$ 600.000,00$

$\$ 500.000,00$

$\$ 400.000,00$

$\$ 300.000,00$

$\$ 200.000,00$

$\$ 100.000,00$

$\$ 0,00$
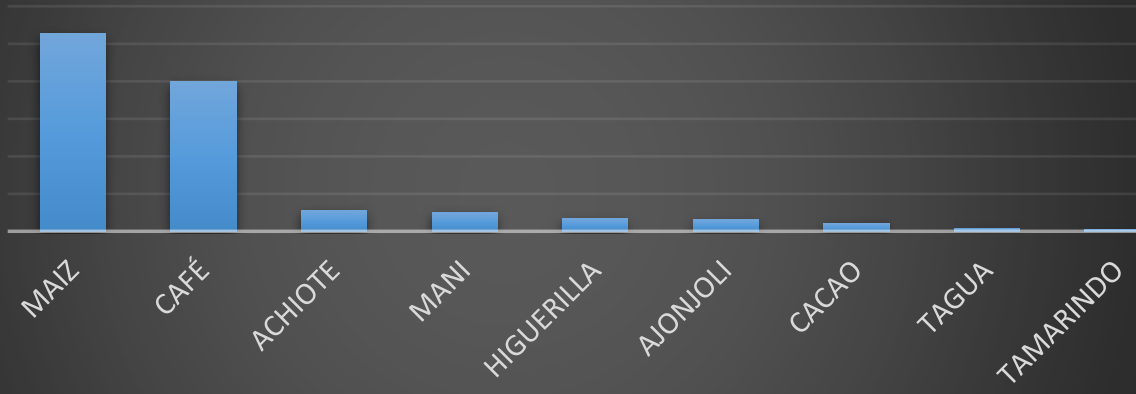

IVENTAS AÑO 2020 EN DÓLARES

Fuente: Autoría propia.

En ese sentido, el marketing digital o e-marketing se refiere a aquellas estrategias y técnicas que utilizan métodos en línea para llegar a los clientes objetivo (Bhosage, Mr, 2018). También se denomina marketing en red, marketing en red, marketing digital o marketing en línea. En general, se refiere al proceso de marketing de productos 0 servicios mediante el uso de Internet.
El marketing incluye no solo el marketing en red, sino también el marketing a través del correo electrónico y los medios locales. La comercialización electrónica de productos agrícolas se refiere a la comercialización de productos agrícolas a través de Internet a través de empresas agrícolas profesionales $\circ$ consumidores finales.

Tabla 2. Ventas y pronósticos de las bodegas de Jipijapa.

\begin{tabular}{|l|r|r|}
\hline $\begin{array}{c}\text { PRODUCTOS } \\
\text { AGRICOLAS }\end{array}$ & $\begin{array}{c}\text { VENTAS AÑO 2020 } \\
\text { EN DÓLARES }\end{array}$ & $\begin{array}{l}\text { PRONÓSTICO DE } \\
\text { VENTAS CON NUEVAS } \\
\text { ESTRATEGIAS }\end{array}$ \\
\hline MAIZ & $\$ 528.000,00$ & $\$ 580.800,00$ \\
\hline CAFÉ & $\$ 400.000,00$ & $\$ 440.000,00$ \\
\hline ACHIOTE & $\$ 56.000,00$ & $\$ 61.600,00$ \\
\hline MANI & $\$ 35.000,00$ & $\$ 55.000,00$ \\
\hline HIGUERILLA & $\$ 32.000,00$ & $\$ 38.500,00$ \\
\hline AJONJOLI & $\$ 22.000,00$ & $\$ 35.200,00$ \\
\hline CACAO & $\$ 7.000,00$ & $\$ 24.200,00$ \\
\hline TAGUA & $\$ 4.200,00$ & $\$ 7.700,00$ \\
\hline TAMARINDO & $\$ 1.134 .200,00$ & $\$ 4.620,00$ \\
\hline TOTAL, VENTAS & & $\$ 1.247 .620,00$ \\
\hline
\end{tabular}

Fuente: Autoría propia. 
Así como existen mercados físicos para productos agrícolas, también existen mercados en línea para productos agrícolas. Con la proliferación de mercados en línea, se ha vuelto más fácil vender productos en línea. Hay muchos mercados en línea para agricultores en Ecuador. Algunos ejemplos son proveedores independientes, mercados de agricultores en línea, etc. Enumerar los productos agrícolas en estas plataformas es otra forma de obtener más exposición y ventas posteriores.

\subsection{Beneficios del marketing electrónico para los agricultores}

Amplio mercado: Los agricultores pueden vender sus productos en todo el mundo. Cubre una gran cantidad de clientes de diferentes estados y también de diferentes países.

Mercado continuo: El mercado en línea está abierto las 24 horas del día, por lo que los agricultores pueden vender sus productos en cualquier momento y cuando lo deseen.
Comprador adecuado y precio adecuado: los agricultores deben colocar sus productos en línea. precio adecuado para sus productos, entonces tienen la posibilidad de vender sus productos a los compradores adecuados.

Menos costo: no hay intermediarios, por lo que hay un costo menor o, a veces, sin costo. Sin desperdicio de productos agrícolas, la mayoría de los productos agrícolas serán destruidos debido a la falta de disponibilidad de los clientes a tiempo. Dado que el mercado online es un mercado continuo, no hay problema de desperdicio de ningún producto.

\subsection{Medios digitales para la venta de productos agrícolas}

\section{Tienda web personal}

Tener su propia tienda web es la mejor manera en línea de vender sus productos agrícolas en comparación con vender sus productos agrícolas en las redes sociales, una tienda de comestibles en línea y un mercado en línea, porque tener una tienda web personal le permite al comerciante controlar de cerca sus 
productos agrícolas ventas en su plataforma.

\section{Entrega de comida (Delivery):}

Al procesar productos agrícolas en forma comestible, se pueden vender en línea a través de un servicio de entrega de alimentos. Hay muchos restaurantes que entregan comida a los clientes cuando los clientes piden comida en línea. La entrega de alimentos generalmente se realiza a través de sitios de compra de alimentos en línea tales como Glovo o Uber Eats.

\section{Conclusiones}

En la etapa actual de desarrollo, los servicios digitales que ofrecen diversos bienes y servicios son cada vez más populares y demandados entre la población. Para los pequeños y medianos comercios, existen dificultades para ingresar al mercado, debido al volumen de su producción. Para los operadores de red y las grandes instalaciones minoristas, es más preferible cooperar con los grandes productores del complejo agroalimentario, que pueden garantizar el suministro de sus productos de acuerdo con los volúmenes requeridos. A su vez, la cooperación de los productores con las empresas de procesamiento y los intermediarios de ventas conduce a un aumento en el costo de los bienes para el usuario final. Uno de los factores importantes es la disponibilidad de productos agrícolas para la población. En síntesis, las tecnologías digitales deben implementarse en todas las áreas de actividad, lo que mejorará la calidad de vida de la población y la competitividad de la economía del cantón Jipijapa y a nivel internacional. La pandemia del coronavirus COVID-19 destacó la importancia de introducir y desarrollar servicios digitales en la comercialización de productos agrícolas.

\section{Bibliografía}

AB, Vijayakumar. (2019). Farmer's insight towards marketing of Agriculture products. Vol 1. 2581-6748.

Bhosage, Mr. (2018). E-Marketing of Agricultural Products. International Journal of Trend in Scientific Research and Development. Special Issue. 70-73. 10.31142/ijtsrd18675. 
Bhosage, Mr. (2018). E-Marketing of Agricultural Products. International Journal of Trend in Scientific Research and Development. Special Issue. 70-73. 10.31142/ijtsrd18675.

Bose, Subhas Chandra \& Kiran, Ravi. (2021). Digital Marketing: A Sustainable Way to Thrive in Competition of Agriculture Marketing. 10.1007/978-981-33-47915_8.

Das, Sreelakshmi. (2019). The Role of Women in Production and Marketing of Agricultural Products. International Journal for Research in Applied Science and Engineering Technology. 7. 411-415. 10.22214/ijraset.2019.8057.

FA, SHAH. (2021). Losses in the marketing of agricultural products. XF2006107557.

Fink, Günther \& Jack, B. \& Masiye, Felix. (2020). Seasonal Liquidity, Rural Labor Markets, and Agricultural Production. American Economic Review. 110. 33513392. 10.1257/aer.20180607.

Kajale, Jayanti \& Shroff, Sangeeta. (2021). Women in Agriculture. Review of Market Integration. 097492922110188. $10.1177 / 0974929221101887$ 9.
Krizhanovskaya, I. (2014). Market of Agricultural Products and its Regulation. The Advanced Science Journal. 2014. 99102.

10.15550/ASJ.2014.12.099.

Osinina, Alexandra. (2021). THE FEATURES OF INTERNATIONAL MARKETING OF AGRICULTURAL PRODUCTS. Economy, labor, management in agriculture. 108-117. $10.33938 / 212-108$

Ploeg, Jan. (2019). Labor, Markets, and Agricultural Production. $10.1201 / 9780429043222$.

Razorvin, Ivan \& Usova, N. \& Loginov, Mihail. (2021). Increasing the availability of agricultural products for the population through the development of digital marketing services. Agrarian Bulletin of the. 79-85. 10.32417/1997-4868-202113-79-85.

SNI. (2015). Obtenido de: http://app.sni.gob.ec/snilink/sni/PORTAL_SNI/data_si gad_plus/sigadplusdocument ofinal/1360000630001_3_1603-2015_21-28-25.ECONOMICO\%20PRODUCT IVO 